\title{
The hadron spectrum from twisted mass QCD with a strange quark
}

\author{
Abdou M. Abdel-Rehim ${ }^{a}$, Randy Lewis ${ }^{* a}$ and R. M. Woloshyn ${ }^{b}$ \\ ${ }^{a}$ Department of Physics, University of Regina, Regina, SK, Canada, S4S OA2 \\ ${ }^{b}$ TRIUMF, 4004 Wesbrook Mall, Vancouver, BC, Canada, V6T 2A3 \\ E-mail: randy.lewis@uregina.ca
}

\begin{abstract}
Various suggestions exist for incorporating the strange quark into twisted mass QCD. One option for quenched simulations is to employ two twisted doublets, $(u, d)$ and $(c, s)$, with separate twist angles. Working in the isospin limit, preliminary results for the quenched spectrum of strange hadrons are presented, with both twist angles tuned to $\pi / 2$. Splittings within the mass multiplets provide some insight into the symmetry breaking effects of twisted mass lattice QCD.
\end{abstract}

XXIIIrd International Symposium on Lattice Field Theory

25-30 July 2005

Trinity College, Dublin, Ireland

\footnotetext{
*Speaker.
} 


\section{Motivation}

Twisted mass lattice QCD (tmLQCD) offers an efficient mechanism for eliminating unphysical zero modes[1] and removing $O(a)$ errors[2] from Wilson simulations. Given the successes of tmLQCD in dealing with the $(u, d)$ quark doublet, it now remains to extend the theory to include other quark flavours, noting that the next lightest pair, $(c, s)$, is far from being degenerate. The present work makes a step in this direction by investigating one option.

Some unphysical flavour symmetry violation occurs at non-zero lattice spacings in tmLQCD. For example, in the two flavour theory with a degenerate $(u, d)$ doublet,[3, 4$]$

$$
\left(m_{\Delta^{++}}=m_{\Delta^{-}}\right) \neq\left(m_{\Delta^{0}}=m_{\Delta^{+}}\right) .
$$

When tmLQCD is extended to include the strange quark, many splittings arise among strange hadrons as well. Since all unphysical flavour splittings must vanish in the continuum limit, they provide a measure of discretization errors in tmLQCD.

\section{Lattice action}

There is more than one way to incorporate the strange quark into tmLQCD. In the present work, we use a tmLQCD fermion action similar to that of Ref. [5] which is a four-quark action, though the fourth (charm) quark will not affect any of our quenched three-flavour spectrum computations. The action is

$$
S_{f}[\psi, \bar{\psi}, U]=a^{4} \sum_{x} \bar{\psi}(x)\left(M+i \mu \gamma_{5}+\gamma \cdot \nabla^{ \pm}-\frac{a}{2} \sum_{v} \nabla_{v}^{*} \nabla_{v}\right) \psi(x),
$$

where

$$
\begin{aligned}
\psi^{T} & =(u, d, c, s), \\
M & =\operatorname{diag}\left(M_{l}, M_{l}, M_{c}, M_{s}\right), \\
\mu & =\operatorname{diag}\left(\mu_{l},-\mu_{l}, \mu_{c},-\mu_{s}\right) .
\end{aligned}
$$

The parameters $M_{n}$ and $\mu_{n}$ denote the standard quark masses and twisted quark masses respectively. Forward $(\nabla)$, backward $\left(\nabla^{*}\right)$ and symmetric $\left(\nabla^{ \pm}\right)$derivatives are standard. To obtain automatic $O(a)$ improvement we choose maximal twist. The classical definition would be

$$
\begin{aligned}
& \omega_{1} \equiv \arctan \left(\frac{\mu_{l}}{M_{l}}\right)=\frac{\pi}{2}, \\
& \omega_{2} \equiv \arctan \left(\frac{\mu_{c}}{M_{c}}\right)=\arctan \left(\frac{\mu_{s}}{M_{s}}\right)=\frac{\pi}{2},
\end{aligned}
$$

but quantum corrections lead to renormalization of both $M$ and $\mu$. Maximal twist is therefore defined nonperturbatively by[3, 6, 7]

$$
\tan \omega_{n}=\left(\frac{i \sum_{\vec{x}}\left\langle\tilde{V}_{v}^{-}(\vec{x}, t) P^{+}(0)\right\rangle}{\sum_{\vec{x}}\left\langle\tilde{A}_{v}^{-}(\vec{x}, t) P^{+}(0)\right\rangle}\right)_{n}
$$

where a tilde denotes currents constructed from fields in the twisted basis. The correlators on the right hand side of Eq. (2.7) require only a single fermion propagator, hence the single subscript $n$. In the present work, this fermion action will be combined with the standard Wilson gauge action. 


\begin{tabular}{cccccl}
\hline$\beta$ & \#sites & \#configs & $a M_{q}$ & $a \mu_{q}$ & physical mass \\
\hline 5.85 & $20^{3} \times 40$ & 594 & -0.8965 & 0.0376 & $\sim m_{s}$ \\
& & & -0.9071 & 0.0188 & $\sim m_{s} / 2$ \\
& & & -0.9110 & 0.01252 & $\sim m_{s} / 3$ \\
6.0 & \multirow{2}{*}{$20^{3} \times 48$} & \multirow{2}{*}{600} & -0.9150 & 0.00627 & $\sim m_{s} / 6$ \\
& & & -0.8110 & 0.030 & $\sim m_{s}$ \\
& & & -0.8195 & 0.015 & $\sim m_{s} / 2$ \\
& & & -0.8210 & 0.005 & $\sim m_{s} / 3$ \\
\hline
\end{tabular}

Table 1: Parameter choices for the simulations.

\section{Simulation details}

Table 1 shows the parameter values chosen for our action and lattices. The authors of Ref. [8] used $r_{0}$ to determine the lattice spacings: $a=0.123 \mathrm{fm}$ for $\beta=5.85$ and $a=0.093 \mathrm{fm}$ for $\beta=6.0$.

The standard mass parameter values, $a M_{q}$, were determined in Ref. [3] to correspond to maximal twist as defined by Eq. (2.7). The twisted mass parameters range from the approximate physical strange quark mass, $m_{s}$, to $1 / 6$ of $m_{s}$. Notice that the standard mass parameter is tuned for each individual twisted mass parameter so, for example, the quark and anti-quark in a computation of the kaon mass will have separate $a M_{q}$ values.

Local interpolating operators are used throughout this work, and three state fits to the resulting correlators are performed.

\section{Results for meson masses}

Numerical results for ground state pseudoscalar meson and vector meson masses are displayed for $\beta=6.0$ in Fig. 1. (Comparable results were obtained for $\beta=5.85$.) All quark/anti-quark mass combinations from Table 1 are shown in this figure, with arrows indicating the values of twisted quark masses (i.e. the horizontal axis of each plot) corresponding to the physical hadrons. For pseudoscalar mesons, significant mass splittings are evident between charged and neutral mesons, as read from the vertical axis of each plot.

The observed mass splitting between $\pi^{ \pm}$and $\pi^{0}$ is affected both by quenching and by our omission of "disconnected" contributions. (See Ref. [9] for a recent tmLQCD study of this splitting.) However, the $K^{ \pm}$to $K^{0}, \bar{K}^{0}$ splitting requires no disconnected contributions and thus our data provide the complete quenched result in that case. Given the automatic $O(a)$ improvement of masses in tmLQCD[2], this unphysical kaon splitting should vanish linearly with $a^{2}$. Figure 2 shows that our two $\beta$ values are consistent with this expectation.

In contrast to the pseudoscalar mesons, Fig. 11 shows no significant splitting between charged and neutral $K^{*}$ mesons within the statistical uncertainties.

\section{Results for baryon masses}

The lack of flavour symmetry in tmLQCD (with $a \neq 0$ ) means the physical octet of spin-1/2 

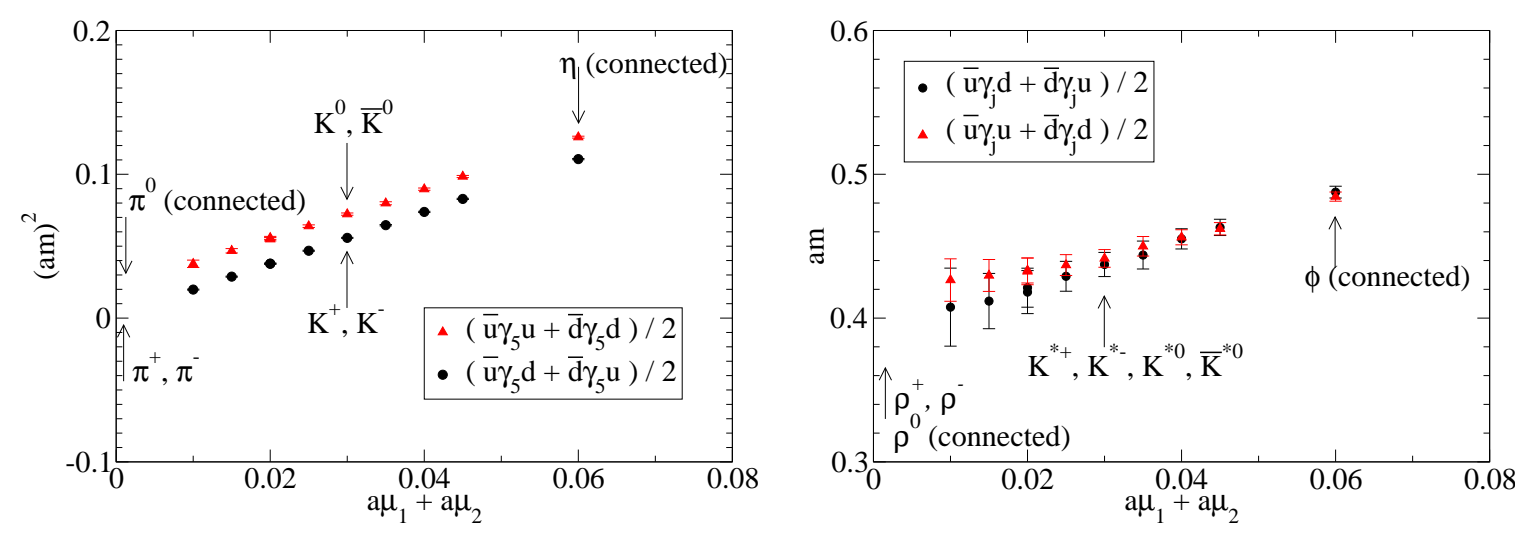

Figure 1: Pseudoscalar (left panel) and vector (right panel) meson masses as functions of the sum of quark and anti-quark twisted masses at $\beta=6.0$.

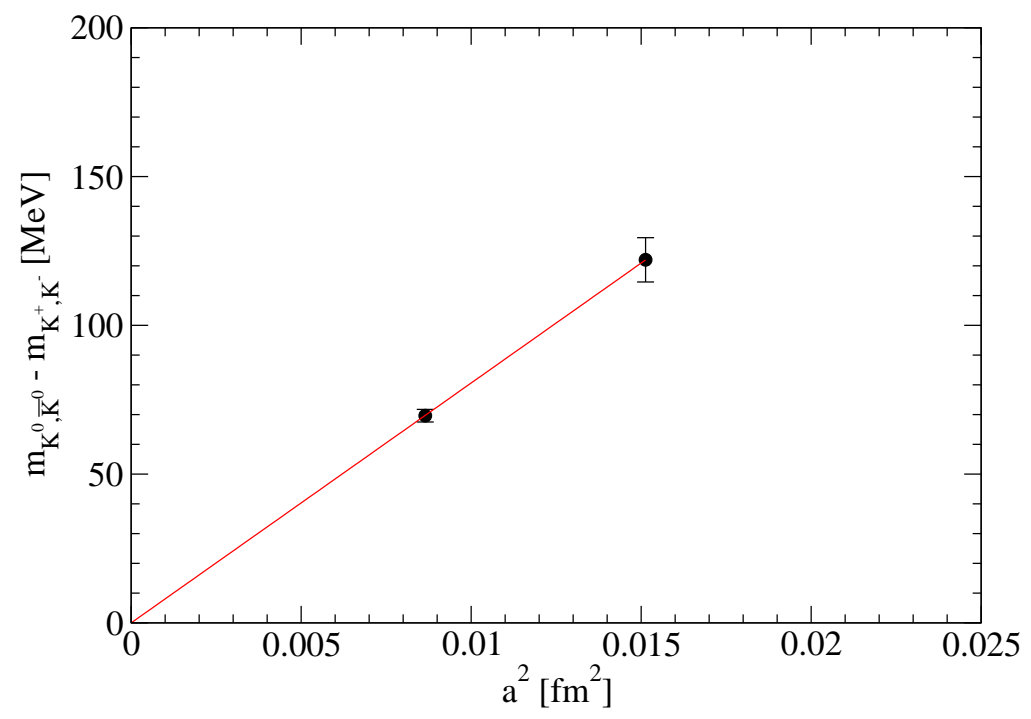

Figure 2: Mass splitting between neutral and charged kaons as a function of squared lattice spacing.

baryons has no exact mass degeneracies and the decuplet of spin-3/2 baryons has just two:[3, 那

$$
\begin{aligned}
m_{\Delta^{++}} & =m_{\Delta^{-}}, \\
m_{\Delta^{0}} & =m_{\Delta^{+}} .
\end{aligned}
$$

For our numerical study of baryon masses, the local source and sink operators are given in the legends of each panel in Fig. 3. All combinations of quark masses from Table 1 were used at each $\beta$ value for both spin-1/2 and spin-3/2 operators, though Fig. 通 only displays data from one $\beta$ as an example for each spin.

Any flavour splittings among these numerical results for baryons are at the edge of statistical significance. Clarification of these splittings will require better operators and increased statistics.

Figure 4 compares the baryon masses in physical units to the spectrum observed in nature. The tmLQCD results lie systematically above experiment, which could be a quenching artifact and/or 

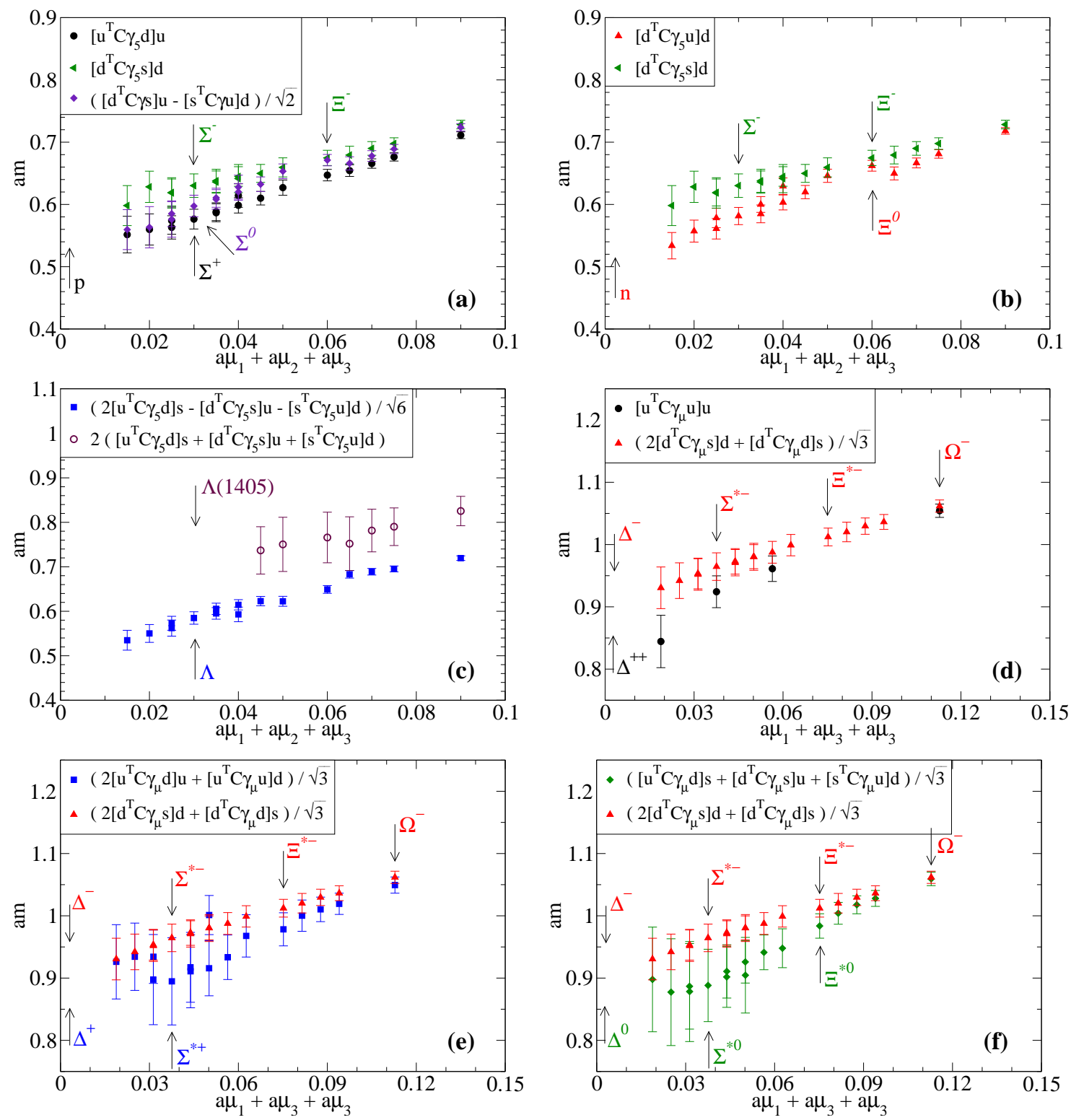

Figure 3: Panels (a), (b) and (c): $(1 / 2)^{+}$octet baryon masses, plus one $(1 / 2)^{-}$channel to discuss the $\Lambda(1405)$, as functions of the sum of the twisted quark masses at $\beta=6.0$. Panels (d), (e) and (f): $(3 / 2)^{+}$ decuplet baryon masses as functions of the sum of the twisted quark masses at $\beta=5.85$.

a consequence of extracting the lattice spacing from $r_{0}$. The two $\beta$ values produce essentially equivalent results, suggesting that we are already in the scaling region.

\section{Conclusions}

One particular method for incorporating the strange quark into tmLQCD has been explored by directly computing the hadron mass spectrum. This approach to 3-flavour tmLQCD produces a large splitting among kaon masses at $a \gtrsim 0.1 \mathrm{fm}$. The splitting vanishes linearly with $a^{2}$ as 


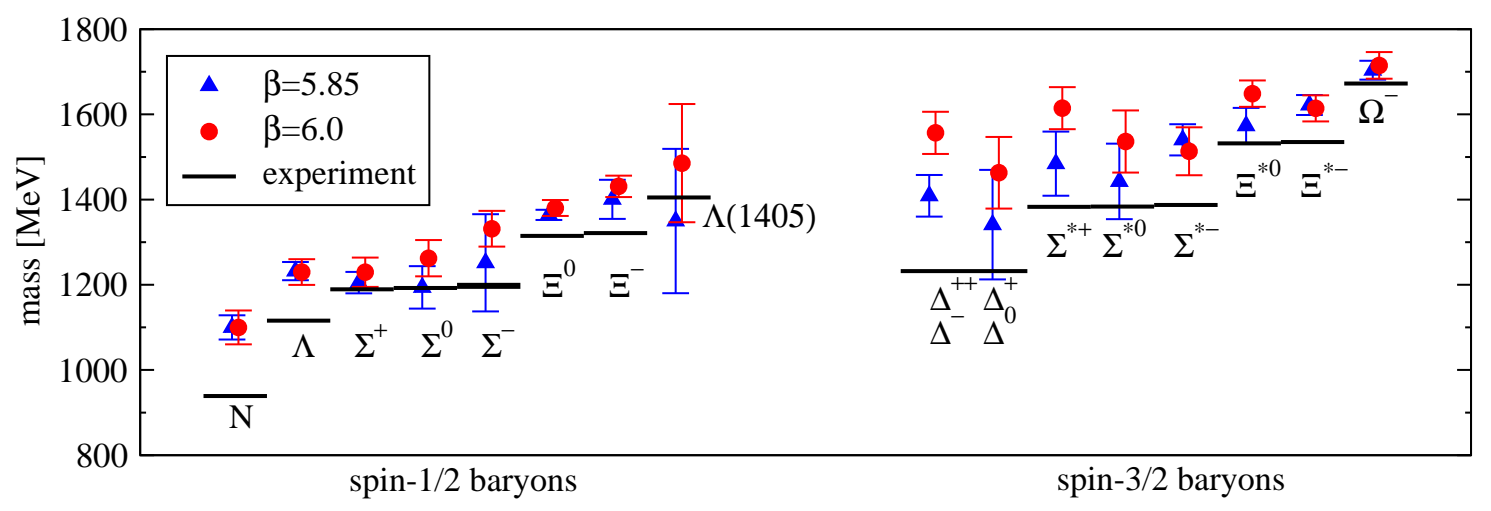

Figure 4: The quenched baryon spectrum compared to experiment.

$a \rightarrow 0$ as expected. Some of the anticipated baryon splittings are potentially discernable, though small. Because the fermion determinant is complex, this method is better suited for quenched simulations than for dynamical ones. Other options for tmLQCD with a strange quark should also be investigated and compared.

\section{Acknowledgements}

This work was supported in part by the Natural Sciences and Engineering Research Council of Canada, the Canada Foundation for Innovation, the Canada Research Chairs Program and the Government of Saskatchewan.

\section{References}

[1] R. Frezzotti, P. A. Grassi, S. Sint and P. Weisz [Alpha collaboration], JHEP 0108, 058 (2001) [arXiv:hep-lat/0101001].

[2] R. Frezzotti and G. C. Rossi, JHEP 0408, 007 (2004) [arXiv:hep-lat/03060114].

[3] A. M. Abdel-Rehim, R. Lewis and R. M. Woloshyn, Phys. Rev. D 71, 094505 (2005) [arXiv:hep-lat/0503007].

[4] A. Walker-Loud and J. M. S. Wu, Phys. Rev. D 72, 014506 (2005) [arXiv:hep-lat/0504001].

[5] C. Pena, S. Sint and A. Vladikas, JHEP 0409, 069 (2004) [arXiv:hep-lat/ 04050028 ].

[6] F. Farchioni et al., Eur. Phys. J. C 42, 73 (2005) [arXiv:hep-lat/0410031].

[7] S. R. Sharpe and J. M. S. Wu, Phys. Rev. D 71, 074501 (2005) [arXiv:hep-lat/0411021].

[8] K. Jansen, A. Shindler, C. Urbach and I. Wetzorke [XLF Collaboration], Phys. Lett. B 586, 432 (2004) [arXiv:hep-lat/0312013.

[9] F. Farchioni et al., [arXiv:hep-lat/0509036]. 